\title{
LongGF: computational algorithm and software tool for fast and accurate detection of gene fusions by long-read transcriptome sequencing
}

\author{
Qian Liu' ${ }^{1 \dagger}, \mathrm{Yu} \mathrm{Hu}{ }^{1+}$, Andres Stucky², Li Fang ${ }^{1}$, Jiang F. Zhong ${ }^{2}$ and Kai Wang ${ }^{1,3^{*}}$ (D)
}

From The International Conference on Intelligent Biology and Medicine (ICIBM) 2020

Virtual. 9-10 August 2020

\begin{abstract}
Background: Long-read RNA-Seq techniques can generate reads that encompass a large proportion or the entire mRNA/cDNA molecules, so they are expected to address inherited limitations of short-read RNA-Seq techniques that typically generate $<150 \mathrm{bp}$ reads. However, there is a general lack of software tools for gene fusion detection from long-read RNA-seq data, which takes into account the high basecalling error rates and the presence of alignment errors.

Results: In this study, we developed a fast computational tool, LongGF, to efficiently detect candidate gene fusions from long-read RNA-seq data, including CDNA sequencing data and direct mRNA sequencing data. We evaluated LongGF on tens of simulated long-read RNA-seq datasets, and demonstrated its superior performance in gene fusion detection. We also tested LongGF on a Nanopore direct mRNA sequencing dataset and a PacBio sequencing dataset generated on a mixture of 10 cancer cell lines, and found that LongGF achieved better performance to detect known gene fusions over existing computational tools. Furthermore, we tested LongGF on a Nanopore cDNA sequencing dataset on acute myeloid leukemia, and pinpointed the exact location of a translocation (previously known in cytogenetic resolution) in base resolution, which was further validated by Sanger sequencing.

Conclusions: In summary, LongGF will greatly facilitate the discovery of candidate gene fusion events from longread RNA-Seq data, especially in cancer samples. LongGF is implemented in C++ and is available at https://github. com/WGLab/LongGF.
\end{abstract}

Keywords: Gene fusion, Long-read sequencing, Transcriptome sequencing, Computational tool

\footnotetext{
* Correspondence: wangk@email.chop.edu

${ }^{\dagger}$ Qian Liu and Yu Hu contributed equally to this work.

${ }^{1}$ Raymond G. Perelman Center for Cellular and Molecular Therapeutics, Children's Hospital of Philadelphia, Philadelphia, PA 19104, USA

${ }^{3}$ Department of Pathology and Laboratory Medicine, Perelman School of

Medicine, University of Pennsylvania, Philadelphia, PA 19104, USA

Full list of author information is available at the end of the article
}

(c) The Author(s). 2020 Open Access This article is licensed under a Creative Commons Attribution 4.0 International License, which permits use, sharing, adaptation, distribution and reproduction in any medium or format, as long as you give appropriate credit to the original author(s) and the source, provide a link to the Creative Commons licence, and indicate if changes were made. The images or other third party material in this article are included in the article's Creative Commons licence, unless indicated otherwise in a credit line to the material. If material is not included in the article's Creative Commons licence and your intended use is not permitted by statutory regulation or exceeds the permitted use, you will need to obtain permission directly from the copyright holder. To view a copy of this licence, visit http://creativecommons.org/licenses/by/4.0/ The Creative Commons Public Domain Dedication waiver (http://creativecommons.org/publicdomain/zero/1.0/) applies to the data made available in this article, unless otherwise stated in a credit line to the data. 


\section{Background}

Gene fusion is a process by which two or more distinct genes are fused into a single gene [1]. Gene fusion could be the results of trans-splicing events or structural variants such as chromosomal translocation, interstitial deletion or chromosomal inversion. Gene fusion plays a critical role in transcriptome diversity and may be associated with human diseases, especially cancer. One of the first known gene fusions was reported to induce chronic myeloid leukemia [2], and since then, more and more gene fusions have been found to play a critical role in tumorigenesis [3-5], such as Ewing's sarcoma and synovial sarcoma $[6,7]$, prostate cancer [8], breast cancer [9], bladder cancer [10], colorectal cancer [11], ovarian cancer [12], lung cancer [13] and tumors in central nervous systems $[14,15]$. Importantly, gene fusions can be used as biomarkers for cancer diagnosis, such as in breast cancer [16] and ovarian cancer [17], and also used as therapeutic targets for cancer [18-21]. The ability to target and better understand gene fusions may lead to the development of novel targeted therapies in the future.

Gene fusions at a transcriptome-wide scale can be detected using RNA-seq techniques, and tens of computational methods have already been developed for this purpose on short-read RNA-seq data, including alignment-based and assembly-based approaches. Alignment-based methods detect gene-fusions on short reads mapped to annotated reference genome or transcriptome, such as Arriba [22], ChimeraScan [23], ChimPipe [24], deFuse [25], FusionCatcher [26], FusionHunter [27], FusionMap [28], FusionQ [29], FusionScan [30], InFusion [31], MapSplice [32], PRADA [33], SnowShoesFTD [34], SOAPfuse [35], Star-fusion [36], STARChip [37], STAR-SEQR (https://github.com/ExpressionAnalysis/STAR-SEQR), and Tophat-fusion [38]. Assemblybased methods, such as BreakFusion [39], EricScript [40], Fusion-Bloom [41], FuSeq [42], JAFFA [43], NeoFuse [44], nFuse [45], Pizzly [46] and ShortFuse [47], predict genefusions by identifying break points using assembly sequences from short-reads. Several review studies have assessed different methods on both simulation data and real short-read RNA-seq data [36, 48, 49], and evaluated the performance in detecting gene fusions. Both alignment- and assembly-based methods require the availability of specific reads in capturing informative transcript sequence to identify fusion points. However, short-read data (typically $<150 \mathrm{bp}$ ) has inherited limitations to detect full length of gene isoforms, suffers from assembly ambiguity, and cannot resolve repetitive regions or lowcomplexity regions. Long-read RNA-seq techniques can generate sequenced reads with tens of thousands of bases, and thus can capture the majority of transcriptional isoforms in single reads without transcriptome assembly.
In the past few years, long-read RNA-Seq techniques are increasingly recognized to improve our understanding of transcriptomic complexity over short-read RNA-Seq. Computational tools designed for long-read RNA-Seq data, such as Mandalorion [50], FLAIR [51] and LIQA [52], can identify novel transcripts and quantify isoform specific expression levels. However, to our knowledge, there are limited available tools to detect gene fusions on long-read RNA-seq data. In this study, we proposed a novel approach called LongGF to detect candidate gene fusion events from long-read RNA-seq data. We examined the performance characteristics of LongGF on a set of simulation data. To further evaluate the real-world utility of LongGF, we tested LongGF on several long-read RNAseq data sets: Oxford Nanopore data (via direct mRNA sequencing) and PacBio data on the universal human reference RNA-seq sample, as well as Nanopore data (via full-length cDNA sequencing) of a patient with acute myeloid leukemia (AML). We compared LongGF against short-read gene fusion detectors, Tophat-Fusion and STAR-Fusion, together with a hybrid method IDPFusion. Our evaluation demonstrated that LongGF successfully detected candidate gene fusions from long-read RNAseq data, and some of these fusions are previously known or can be validated by additional Sanger sequencing.

\section{Methods}

\section{Framework of LongGF}

As shown in Fig. 1 (a), the input of LongGF is a BAM file from a long-read RNA-seq data together with a GTF file containing the definition of known genes and their transcriptional isoforms. The BAM file can be generated by different long-read aligners (minimap2 [53] by default). The output of LongGF is a prioritized list of candidate gene fusions together with their supporting long reads. LongGF has several steps to detect gene fusions from the BAM file: get multiple mapped long reads (i.e., reads that map to multiple genomic locations), obtain candidate gene pairs, find gene pairs with non-random supporting long reads, and output prioritized list of candidate gene fusions ranked by the number of supporting reads.

\section{Get multiple mapped long reads}

Given an input BAM file for a RNA-seq data set, we check each mapped record, and filter those long reads which have no supplementary alignment. In BAM format, a long read may have more than 1 significant alignment records in different genomic positions (as shown in Fig. 1 where both long read 1 and long read 2 have 2 alignment records), and one of them is considered as primary alignment, yet others are considered as supplementary alignments if mapped bases in the long read in this alignment have less overlap with mapped bases in 


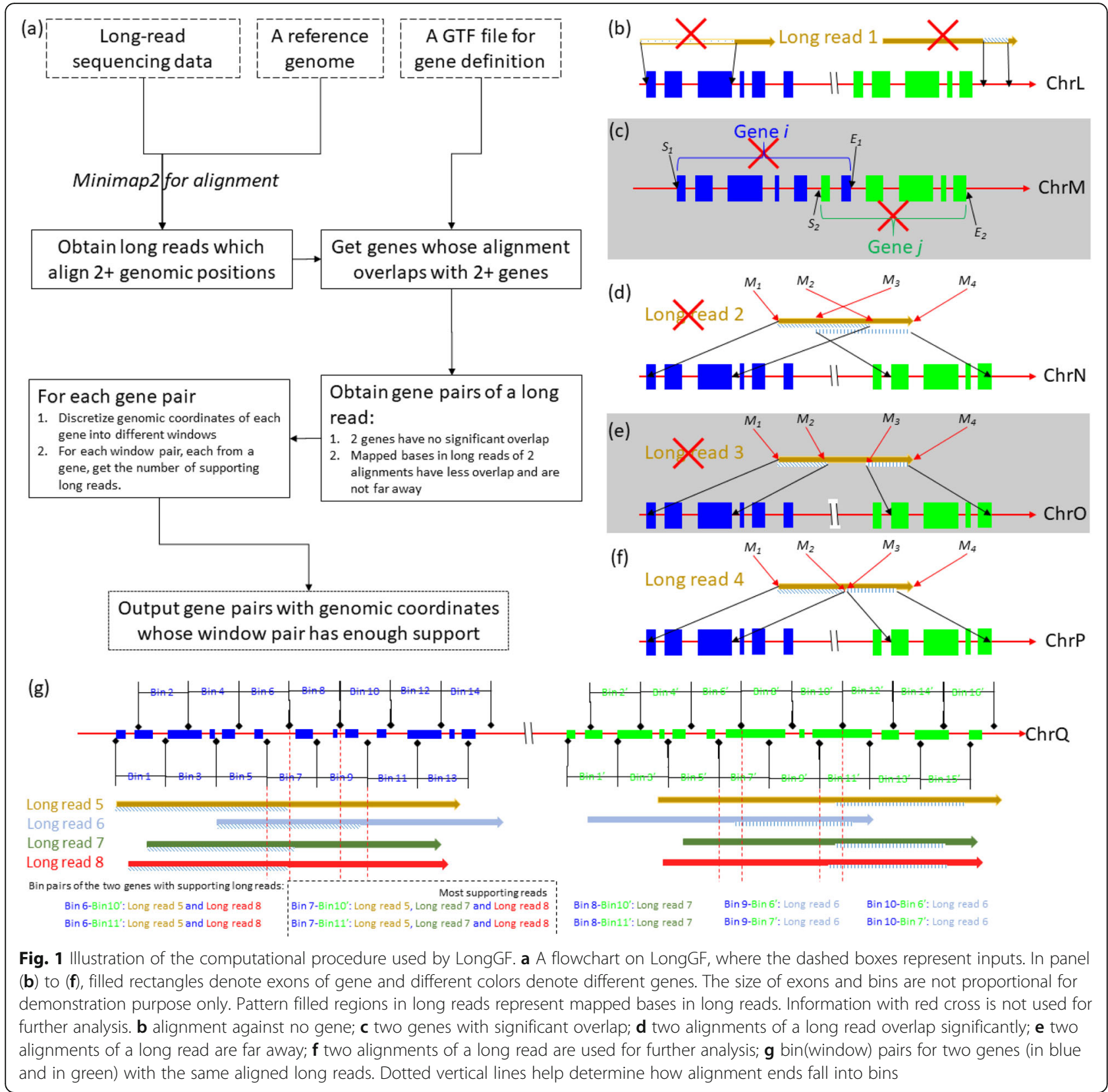

the primary alignment, or as secondary alignment otherwise. Secondary alignments are thus filtered in LongGF, because upstream sequence and downstream sequence around the fusion points of gene fusion are from two independent genes and would have no substantial overlap. Thus, only primary and supplementary alignments are considered in this step.

Obtain candidate gene pairs of multiple mapped long reads For each alignment record for a long read, the mapped genomic positions in a reference genome are compared against a corresponding gene definition with genomic coordinates of exon for each transcript of genes (as shown in Fig. 1 where long reads 2, 3 and 4 are mapped to different genes). If the size of the overlap of the mapped positions and the exons for a transcript is larger than a user-defined threshold, this alignment record is considered to be associated with the transcript. If a long read has more than 1 significant overlap against 2 independent genes, the gene pairs together with the alignment information are summarized; otherwise, it is filtered out as shown in Fig. 1(b). Meanwhile, to filter out noises, some genes or long reads below are not considered: (1) pseudogenes in a GTF gene definition file or two genes whose genomic coordinates have significant overlap: Assume two genes $i$ and $j$ whose starting 
positions are $S_{1}$ and $S_{2}\left(S_{1}<S_{2}\right)$, and ending positions are $E_{1}$ and $E_{2}$, if $E_{1}-S_{2}>0$, the two genes have significant overlap, as shown in Fig. 1 (c). Please note that we do not consider these pseudogenes in the analysis by default, and in LongGF, users can specify whether to use pseudogenes in gene fusion detection; and (2) a long read sequence whose mapped bases of two alignment records do not have an appropriate gap: for example in a long read sequence, the mapped bases from $M_{1}$ to $M_{2}$ are used in one alignment record, and bases from $M_{3}$ to $M_{4}$ are used in another alignment record, $M_{3}>M_{1}$, if $M_{3}-M_{2}$ is larger than a threshold (such as 20, as shown in Fig. 1 (e)) or less than - 20, as shown in Fig. 1 (d), the two alignment records do not have an appropriate gap. In Fig. 1, long reads 2 and 3 are excluded and long read 4 (Fig. 1 (f)) is used for further analysis. Please note that alignments shown in Fig. 1 (c) need users' further investigation for potential gene fusions, while alignments such as those in Fig. 1 (d) and Fig. 1 (e) may indicate complex gene fusions or implicate the presence of potential structural variants.

\section{Find gene pairs with non-random supporting long reads}

Each gene pair generated above is associated with a set of long reads together with their alignment information. Those alignments may not be consistent due to alignment errors or sequencing errors. A consistent support is summarized using the process as shown in Fig. 1 (g): first, aligned genomic positions are discretized into a window with $w$ bp and two adjacent windows have $w / 2$ bp overlap; second, each gene pair is then associated with all possible window pairs; third, for each long read associated with this gene pair, if the fusion points of the two alignment records fall into a window of a gene and into a window of the other gene, the number of supporting long reads for this window pair is increased by 1 ; then, each window pair is associated with the number of supporting long reads together with the fusion points of two alignment records of long reads; fourth, for the window pair with maximum supporting long reads, the averaged genomic position of fusion points is considered as the fusion points of this potential fused gene. By default, one breakpoint is shown in the output for a gene pair, but users can specify the parameters in LongGF to output more breakpoints for each gene pair to facilitate downstream analysis to refine breakpoints.

\section{Output prioritized list of candidate gene fusions}

From a BAM file, multiple candidate gene fusions are detected, and each is associated with a list of a pair of two alignment records on long reads. We rank the potential fused genes according to the number of supporting long reads. More reliable gene fusion events usually have more supporting long reads. We also allow the extraction of reads in specific locations, so that users can easily examine the reads and alignments in visualization tools such as IGV, to visually validate whether the candidate fusion events are reliable.

\section{Datasets for evaluations}

To evaluate the performance, we applied LongGF to several existing long-read RNA-seq data sets using Oxford Nanopore long-read techniques (PRJNA639366 and PRJNA40456 in NCBI Short Read Archive), including one direct mRNA sequencing data set, one full-length cDNA sequencing data set, as well as additional longread RNA-seq data using PacBio sequencing techniques. We also simulated tens of long-read RNA-seq data sets. The description of the datasets is given below.

\section{Long-read sequencing of universal human reference RNA- seq data}

We analyzed two long-read datasets, using Nanopore sequencing and PacBio sequencing, for Universal Human Reference (UHR) RNA which comprises of mixed RNA molecules from a diverse set of 10 cancer cell lines with equal quantities of DNase-treated RNA from adenocarcinoma in mammary gland, hepatoblastoma in liver, adenocarcinoma in cervix, embryonal carcinoma in testis, glioblastoma in brain, melanoma, liposarcoma, histocytic lymphoma in histocyte macrophage, lymphoblastic leukemia and plasmacytoma in B lymphocyte. This reference sample from MicroArray Quality Control [17, 54, 55] project has been utilized in many studies. For example, Gao et al [56] sequenced this UHR RNA sample and treated it as reference to measure the technical variations of scRNA-seq data. Also, the qRT-PCR measurements of gene/isoform expressions from this sample were used to benchmark and optimize computational tools [57-61]. Direct mRNA sequencing protocol was used to generate Nanopore sequencing data, and we used Guppy for basecalling. In total, there are $\sim 476,000$ long reads with $\sim 557$ MB bases. We aligned the Nanopore RNA-seq data against a reference genome (hg38) using minimap2 [53], and 95\% long reads ( $89 \%$ of total bases) were mapped, demonstrating very high sequencing and basecalling quality. Additionally, PacBio has used Iso-seq generated FLNC (full-length non-chimeric) long-read sequencing data for the UHR RNA samples [62]. In total, there are 6,775,127 long reads with $13.7 \mathrm{~GB}$ bases. We aligned PacBio long reads against hg38 using minimap2 [53], and 94\% long reads with 95\% bases were mapped. On the UHR RNA-seq data, the 6 well-known gene fusions used for our benchmarking study on short-read sequencing data include BCAS4-BCAS3, BCR-ABL1, ARFGEF2-SULF2, RPS6KB1TMEM49(VMP1), TMPRSS2-ERG, and GAS6-RASA3. 


\section{Nanopore CDNA sequencing of a patient with AML (acute myeloid leukemia)}

AML is a type of cancer where abnormal myeloblasts are made by bone marrow. Full-length double-stranded cDNA were generated from total RNA by 1D strandswitching RT protocol and the cDNA sample was sequenced using GridION Nanopore sequencer with Guppy basecalling. In total, there are 8,061,683 long reads with $6.6 \mathrm{~GB}$ bases. We aligned the data against a reference genome (hg38) using minimap2 [53], and 63\% long reads ( $73 \%$ bases) were mapped, indicating moderate sequencing and basecalling quality. There is a gene fusion between RUNX1T1 and RUNX1 in this patient from previous cytogenetic studies, but with unknown genomic positions of the breakpoints.

\section{Simulation of long-read RNA-seq data}

We simulated tens of long-read RNA-seq data sets to evaluate the performance of LongGF for gene-fusion detection based on RefSeq gene annotation. To simulate a realistic dataset with known gene fusions, we used NanoSim to generate long-read RNA-seq data [63]. NanoSim simulator program captures the technologyspecific features of long-read data and allows for adjustments upon improvement of Nanopore sequencing technology. The use of NanoSim facilitates the evaluation of LongGF in gene-fusion detection under a realistic setting. To simulate Nanopore RNA-seq reads using NanoSim, the human reference genome sequence (hg19, NCBI build 37) was downloaded from UCSC Genome Browser (https://genome.ucsc.edu/). We characterized parameters for NanoSim using an existing datasets generated from human reference RNA samples. We simulated 10 Nanopore RNA-seq samples (500,000 reads per sample). To make our simulated datasets more realistic, for each sample, we included 100 gene-fusions and assigned expressions to them based on gene expression distribution of a real RNA-seq dataset (expression TPM: 50 gene-fusions > 1000; 50 genefusions ranges from 10 to 1000). Specifically, for each genefusion, we first selected two isoform transcripts from two different genes and assigned fusion points randomly to cut each transcript into two parts ( $5^{\prime}$ end and $3^{\prime}$ end parts). Next, we combined the $5^{\prime}$ end and $3^{\prime}$ end parts from two different genes together to construct a simulated genefusion. The expression level of each gene-fusion was calculated based on the average expression between two genes from the UHR data set. Given annotated gene-fusions and expressions, we generated Nanopore reads using NanoSim. These simulated RNA-seq reads were then mapped to the hg19 reference human genome using minimap2 [53]. Then, we analyzed 10 samples respectively to detect gene fusion events, and compared them to the artificially created gene fusions.

\section{Results}

\section{Performance on simulation datasets}

The characteristics of the simulated data are shown in Fig. 2 (a)(b)(c). The median read length is $1091 \mathrm{bp}$ and the average mapping rate across 10 simulated datasets is $99 \%$. The coverage plot of the simulated data is similar to a real study, demonstrating positional biases and fulllength coverage of isoforms. For all underlying gene fusions, the median number of supporting reads is 42 . Since the ground truth is known, these simulated datasets facilitate the performance evaluation of LongGF.

We explored several measures to quantify the accuracy of gene fusion detection by LongGF on simulation datasets. First, we measured the recall (power) of our method by calculating the proportion of correctly predicted gene fusions among known (artificially introduced during simulation) gene fusions. Second, we measured the precision of LongGF by calculating the proportion of correctly predicted gene fusions among all predicted gene fusions. Last, we evaluated the overall performance of LongGF using F1 score which is a weighted average of precision and recall values ( $\mathrm{F} 1$ score $\left.=2 \cdot \frac{\text { precision-recall }}{\text { precision }+ \text { recall }}\right)$.

Figure 2 (d) (e) shows the distribution of supporting read counts at different events (true positive, false positive) and the summary statistics of LongGF (supporting read detection threshold $\geq 2$ ) based on 10 simulations respectively. 37\% (10 out of 27) of false positives have supporting read count less than 10 . Clearly, LongGF has consistently high precisions (>93\%) across all simulation data sets, indicating that the false positive rate is well controlled. The recall values for LongGF range from 86 to $93 \%$. 3 gene fusions (3\%) were missed by longGF across 10 simulations on average. Most of these false negative gene fusions have low expression (TPM $<50$ on average), leading to limited number of long reads that are mapped to the fusion point between genes. For example, the expression of a missed gene fusion was only 13.2 TPM, and there was no simulated read that is mapped to the fusion point. Accounting for both precision and recall values, F1 scores remain high (> 90\%) for all 10 simulated datasets.

Next, we evaluated the impact of the read coverage on the accuracy of gene fusion detection. We simulated four other datasets with the fusions from the sample with the lowest F1 score (90.8\%) above, and the 5 datasets for this sample have different total read coverages( 2 M, 1 M, 500 $\mathrm{k}, 100 \mathrm{k}$ and $50 \mathrm{k}$ reads respectively). Then, we calculated recall, precision and F1 score for each dataset. As shown in Fig. 2 (f), for datasets with less number of reads, the performance becomes generally lower. Compared to the original dataset with $500 \mathrm{k}$ reads, the performance measurements (recall, precision, F1 score) of $2 \mathrm{M}$ reads were improved by $8.4,2.1$ and $5.7 \%$ respectively. Meanwhile, the measurements (recall, precision, F1 score) dropped by $18.5,10.8$ and $14.5 \%$ when read coverage decreased 

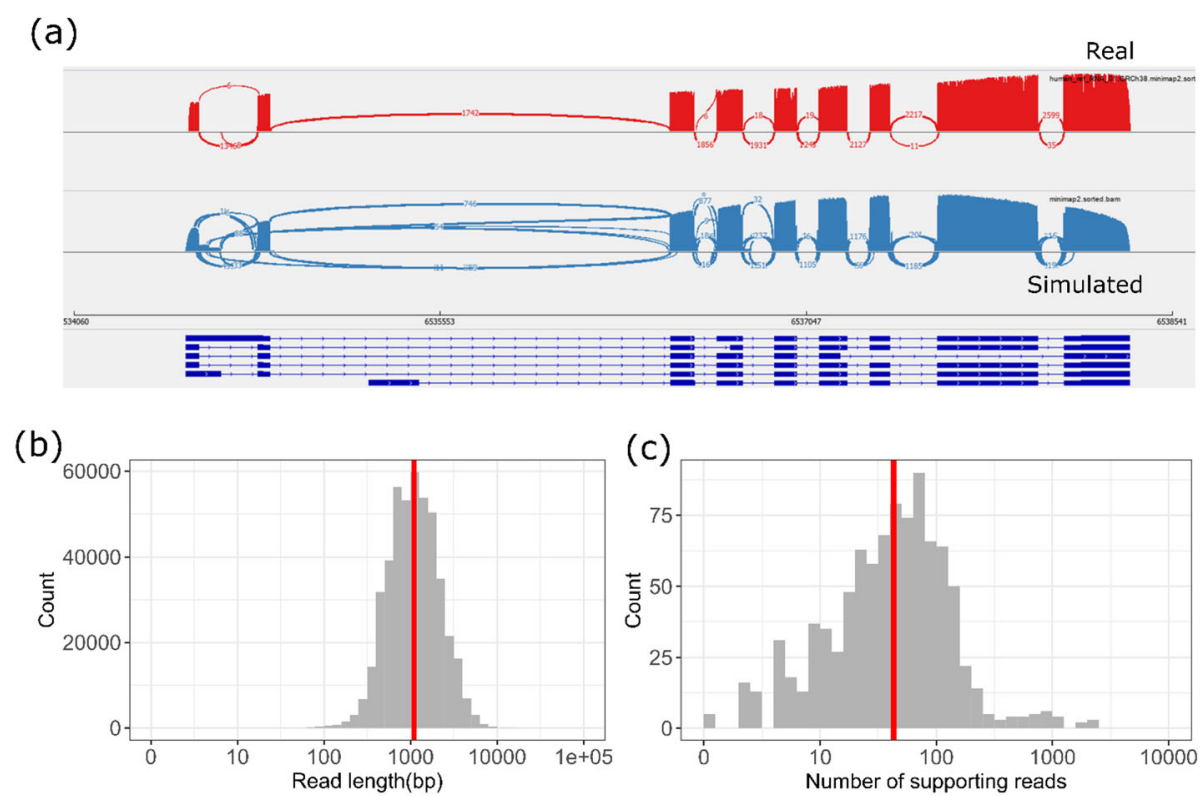

(d)
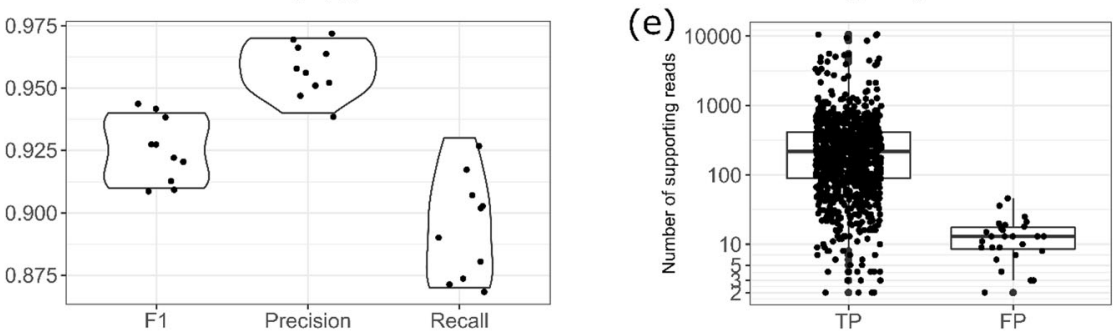

(f)

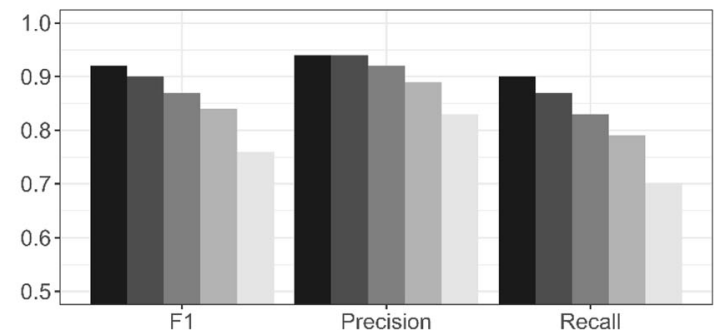

Total read coverage

- $2 \mathrm{M}$

- $1 \mathrm{M}$

- $500 \mathrm{k}$

- $100 \mathrm{k}$

$50 \mathrm{k}$

Fig. 2 Performance evaluation of LongGF from simulation study. a Read coverage plots of UHR data (red) and simulated data(blue) for a random gene. $\mathbf{b}$ Read length distribution from simulated data. $\mathbf{c}$ Distribution of supporting read counts at true gene fusion point from simulated data. $\mathbf{d}$ The measurements (F1, precision and recall) of LongGF based on 10 simulations. e The number of supporting reads at true positive (TF) and false positive (FP). $\mathbf{f}$ The measurements (F1, precision and recall) of LongGF across different total read coverages based on the same simulation dataset

from $500 \mathrm{k}$ to $50 \mathrm{k}$. This is not surprising because on lower coverage data, less number (or none) of the reads are mapped to the fusion breakpoint between two genes, which makes it more difficult for LongGF to detect candidate gene fusion events. Precision is less sensitive to the change of sequencing depth than recall. In summary, LongGF performed robustly (recall: $88.6 \%$, precision: $95.8 \%$, F1 score: $91.9 \%$ in average) in detecting gene fusions using simulation data with $\sim 500 \mathrm{k}$ reads.

\section{Evaluation on the UHR RNA sample by direct mRNA} sequencing

UHR sample contains a mixture of RNA from 10 different cancers and is a widely used benchmarking material to evaluate computational tools for transcriptome analysis. We previously sequenced the sample using Oxford Nanopore direct mRNA sequencing protocols and generated $\sim 476,000$ long reads with $\sim 557 \mathrm{M}$ bases. After aligning the data against hg38 with minimap2 [53], we detected gene fusions using LongGF with the minimum 
Table 1 Candidate gene fusions detected by LongGF on long-read RNA-seq data for universal human reference mRNA sample and for a patient with AML. "A:B" denotes a gene fusion of gene $A$ and gene B. The 6 known gene fusions for 'UHR Nanopore' rows are used for evaluating gene fusion detection, but additional gene fusions may be present for UHR samples

\begin{tabular}{|c|c|c|c|c|c|c|}
\hline \multirow[t]{2}{*}{ Gene fusion } & \multirow[t]{2}{*}{ Datasets } & \multirow{2}{*}{$\begin{array}{l}\text { \#Supporting } \\
\text { reads }\end{array}$} & \multirow{2}{*}{$\begin{array}{l}\text { Read } \\
\text { coverage }\end{array}$} & \multicolumn{2}{|c|}{ Fusion points (hg38 coordinate) } & \multirow[t]{2}{*}{ Benchmark } \\
\hline & & & & Breakpoint 1 & Breakpoint 2 & \\
\hline BCAS4:BCAS3 & \multirow{6}{*}{$\begin{array}{l}\text { UHR } \\
\text { Nanopore }\end{array}$} & 8 & 23 & chr20:50,795,173 & chr17:61,368,325 & Yes \\
\hline MGAT5:IGLC7 & & 7 & 12 & chr2:134,120,290 & chr22:22,922,718 & \\
\hline GAS6:RASA3 & & 2 & 8 & chr13:11,3826,995 & chr13:113,981,855 & Yes \\
\hline ARFGEF2:SULF2 & & 2 & 5 & chr20:48,922,009 & chr20:47,736,942 & Yes \\
\hline AP3D1:JSRP1 & & 2 & 41 & chr19:2,127,153 & chr19:2,252,480 & \\
\hline VMP1:RPS6KB1 & & 2 & 78 & chr17:59,838,294 & chr17:59,910,610 & Yes \\
\hline BCAS4:BCAS3 & \multirow{23}{*}{$\begin{array}{l}\text { UHR } \\
\text { PacBio }\end{array}$} & 206 & 520 & chr20:50795172 & chr17:61368324 & Yes \\
\hline ARFGEF2:SULF2 & & 70 & 389 & chr20:48922011 & chr20:47736941 & Yes \\
\hline FGFR1:NSD3 & & 42 & 53 & chr8:38457533 & chr8:38381797 & $\ln [64]$ \\
\hline LDLR:ZNF333 & & 36 & 51 & chr19:11108452 & chr19:14701590 & $\ln [64]$ \\
\hline SMARCA4:CARM1 & & 28 & 1126 & chr19:10986591 & chr19:10904949 & $\ln [64]$ \\
\hline VMP1:RPS6KB1 & & 26 & 2601 & chr17:59838295 & chr17:59910609 & Yes \\
\hline GAS6:RASA3 & & 22 & 103 & chr13:113826995 & chr13:113981856 & Yes \\
\hline GANAB:B3GAT3 & & 20 & 33 & chr11:62627352 & chr11:62620496 & $\ln [65]$ \\
\hline RPS6KB1:DIAPH3 & & 19 & 2738 & chr17:59930173 & chr13:59666845 & $\ln [64]$ \\
\hline NUP214:XKR3 & & 19 & 39 & chr9:131199013 & chr22:16808083 & $\ln [64]$ \\
\hline MYH6:HOMEZ & & 15 & 27 & chr14:23386622 & chr14:23285911 & $\ln [38]$ \\
\hline PAPOLA:AK7 & & 15 & 78 & chr14:96502599 & chr14:96437835 & $\ln [64]$ \\
\hline CBX3:CCDC32 & & 14 & 26 & chr7:26201744 & chr15:40561981 & $\ln [64]$ \\
\hline MYH9:EIF3D & & 14 & 65 & chr22:36387806 & chr22:36526134 & $\ln [64]$ \\
\hline DCAF6:SEMA4A & & 14 & 885 & chr1:167951860 & chr1:156153370 & \\
\hline ZBTB45:UBE2M & & 11 & 35 & chr19:58518739 & chr19:58557605 & \\
\hline RSBN1:AP4B1 & & 10 & 15 & chr1:113811708 & chr1:113899869 & \\
\hline GCN1:MSI1 & & 10 & 65 & chr12:120190297 & chr12:120347515 & $\ln [64]$ \\
\hline ESR1:CCDC170 & & 9 & 29 & chr6:151702003 & chr6:151573171 & $\ln [64]$ \\
\hline NUP210L:GATAD2B & & 8 & 501 & chr1:154027597 & chr1:153922729 & $\ln [64]$ \\
\hline$A B L 1: B C R$ & & 8 & 298 & chr9:130854065 & chr22:23290408 & Yes \\
\hline ZFP64:ATP1A1 & & 8 & 18 & chr20:52052134 & chr1:116396734 & \\
\hline GOPC:ROS1 & & 8 & 13 & chr6:117566853 & chr6:117321395 & $\ln [64]$ \\
\hline RUNX1T1:RUNX1 & \multirow[t]{2}{*}{ A patient with $\mathrm{AML}$} & 9 & 2373 & chr8:92,017,366 & chr21:34,859,474 & Yes \\
\hline NBEAL1:RPL12 & & 8 & 7244 & chr2:203,190,790 & chr9:127,451,392 & \\
\hline
\end{tabular}

mapped length of $100 \mathrm{bp}$, minimum overlap size of 100 bp between mapped bases and exons of a transcript and $w=50$. The results are shown in Table 1 with the threshold of 2 for minimum supporting long reads. In Table 1, there are the 6 detected gene fusions, and 4 of them are among the 6 known gene fusions on UHR which were previously used for evaluating short-read gene fusion detectors. In particular, the top 1st gene fusion is shown in Fig. 3 in IGV plots where 9 long reads support the gene fusion very well.
Note that it is likely that more than 6 true gene fusions are present in the UHR sample which is composed of 10 different cancers, but we only used the 6 well known fusions for evaluation of LongGF on UHR Nanopore data as the short-read gene fusion detectors did. That is, 2 gene fusions detected by LongGF (possible false positives) are not in the known gene fusion list, and one of them is mapped against a reference region which appears more than 1 times, and thus, this may represent a genuine gene fusion event that was missed by previous 


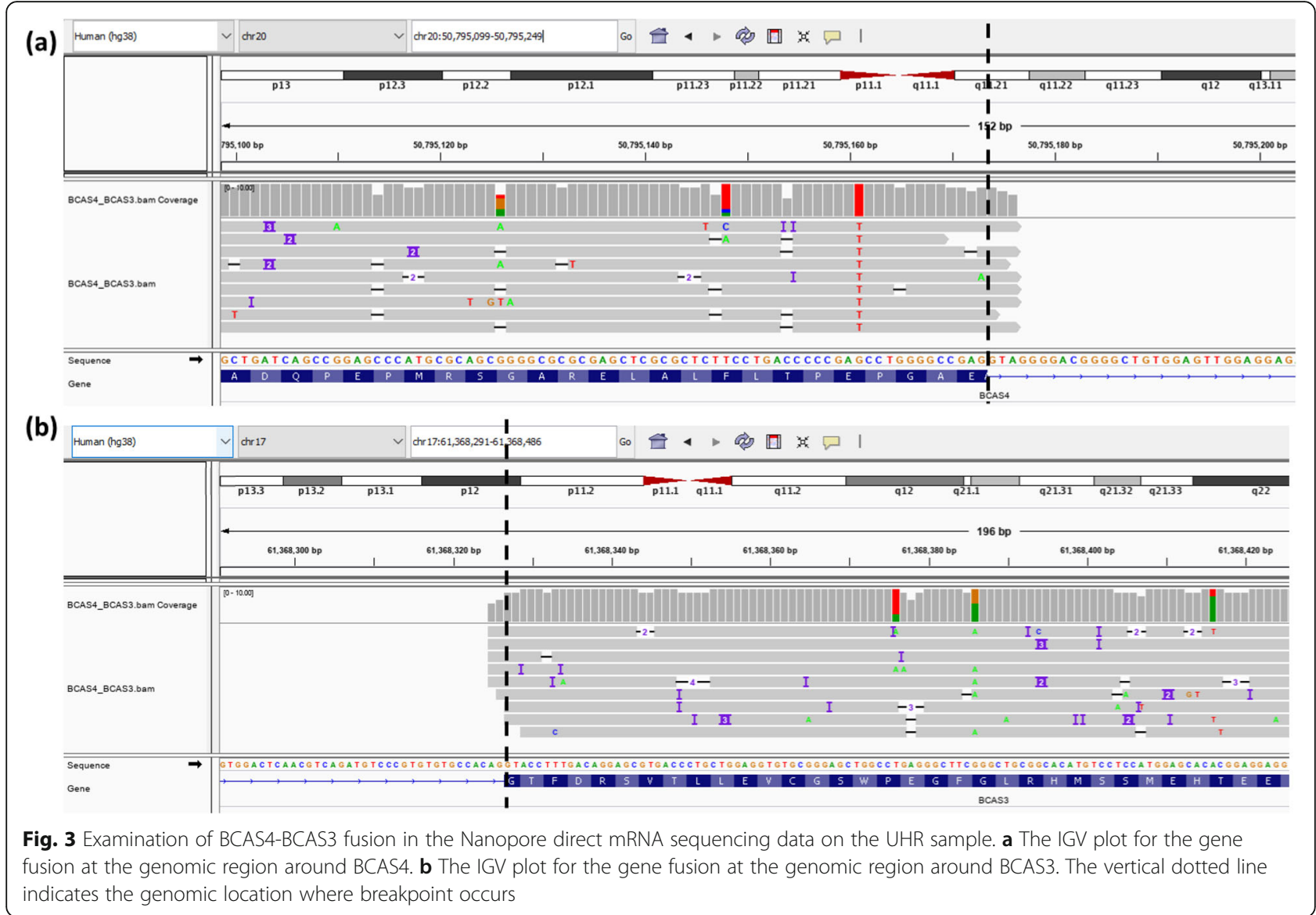

studies. Meanwhile, since 2 false negative events, including the well known BCR-ABL1 gene fusion, are not detected by us, we further examined the sequence data on the genomic region of the BCR-ABL1 gene fusion. We found that the failure to identify BCR-ABL1 gene fusion may be due to the low expression of this fusion in the transcriptome: as shown in short-read RNA-seq data of UHR [38], BCR-ABL1 is $\sim 6$ times less expressed than BCAS4-BCAS3 gene fusion (with 9 supporting long reads in UHR Nanopore RNA-seq data), and $\sim 4$ times less expressed than GAS6-RASA3 (with 2 supporting long reads in UHR Nanopore RNA-seq data) and ARFG EF2-SULF2 gene fusion (with 2 supporting long reads in UHR Nanopore RNA-seq data). Given that the UHR sample is a mixture of 10 different cancer cell lines, it is expected that known gene fusions such as BCR-ABL1 in one cell line will have relatively low allele fraction in the data; therefore, in the long-read RNA-seq data generated by us, we do not have enough coverage on the BCR$\mathrm{ABL} 1$ gene fusion and we were not able to detect this fusion by LongGF.

We further evaluated LongGF on a higher coverage PacBio long-read data on the UHR sample that was sequenced by PacBio [62]. The results were shown in
Table 1. It can be seen from Table 1 that LongGF is able to detect the BCR-ABL1 gene fusion and other known gene fusions detected on the Nanopore data. In particular, the BCR-ABL1 gene fusion only has 8 supporting long reads compared against other known gene fusions (206, 69, 26 and 22 supporting reads for BCAS4-BCAS3, ARFGEF2-SULF2, VMP1-RPS6KB1 and GAS6-RASA3, respectively), which supports our speculation that the low-coverage issue is the reason why LongGF on Nanopore data missed this gene fusion. Additionally, LongGF on the PacBio long-read data detects 23 gene fusions with $>=8$ supporting long reads. We thus checked other detected gene fusions using Mitelman databases (Mitelman databases contains many gene fusions in cancers manually culled from the literature) [64] and other online resources [65]. We found that 19 gene fusions were reported in the literature. In contrast to short-read data, only 3 of top 20 detected gene fusions by STAR-Fusion [36] and 6 of top 20 detected gene fusions by Tophat-Fusion [38] were reported in the literature. Although this is not a direct comparison of the tools to detect gene fusions on long-read and shortread datasets, this analysis suggests that LongGF on longread high-coverage data likely identify more reliable gene fusions with much less false positives. 


\section{Evaluation on a breast cancer dataset}

Moreover, we compared LongGF with IDP-Fusion [66], a gene fusion detector using hybrid data (both long-read and short-read sequencing data), on the MCF-7 breast cancer dataset. Among a set of 71 fusion gene events validated by either PCR and/or Sanger sequencing [66], LongGF and IDP-Fusion detected 25 and 24 events, respectively. The recall of LongGF in detecting fusion genes is comparable to IDP-Fusion on this long-read data on breast cancer, but IDP-Fusion uses both longread and short-read data. LongGF also detected more potential novel gene fusions (ACTB:H3F3B, SLC25A24: NBPF6, STMN1:ACTG1), and these genes were reported to be associated with breast cancer [67-71].
Therefore, compared to hybrid-based gene fusion detector, LongGF yields comparable accuracy for fusion gene detection.

\section{Evaluation on a patient with AML by long-read CDNA sequencing}

To further evaluate the performance of LongGF on real datasets, we analyzed a long-read cDNA sequencing data generated on blood sample from a cancer patient affected with AML. We detected gene fusions using LongGF on this long-read RNA-seq data with the minimum mapped length of $500 \mathrm{bp}$, minimum overlap size of $100 \mathrm{bp}$ between mapped bases and exons of a transcript, and $w=50$. The results are shown in Table 1 with

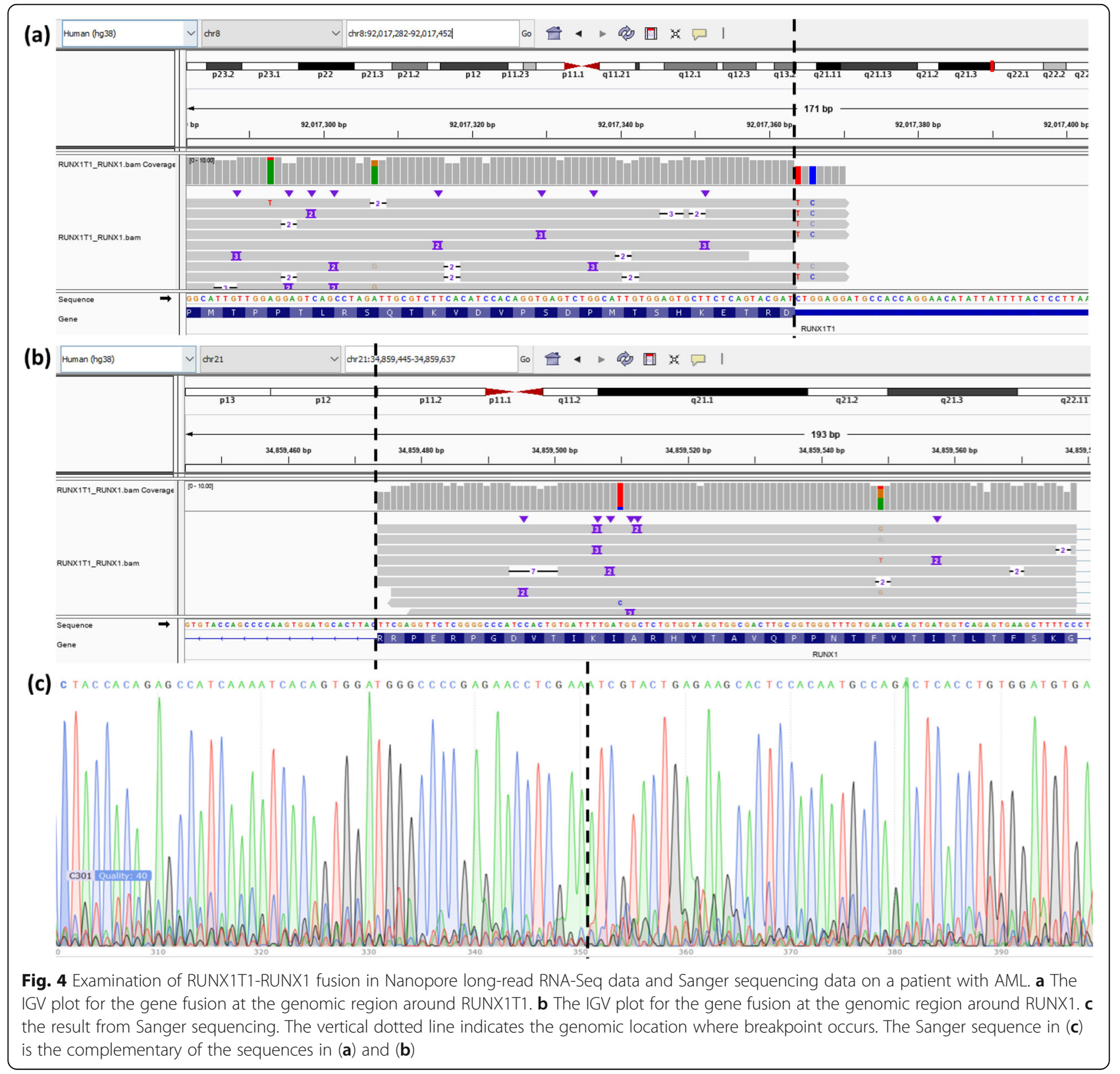


minimum supporting reads of 5 , where 2 gene fusions are detected. One detected gene fusion is RUNX1T1: RUNX1 (as shown in Fig. 4 (a) and (b)), which has been found to be associated with AML [72]. We note that although the same gene fusion was previously known by cytogenetic analysis on this patient, the exact breakpoint location is not known for this patient. We then validated this gene fusion using Sanger sequencing (as shown in Fig. 4 (c)), and we found that the breakpoints of the two genes are chr8:92,017,373 and chr21:34,859,474, respectively. Compared with the results reported by LongGF, the inferred breakpoint at chr21 is exactly the same as the Sanger sequencing result, yet the inferred breakpoints at chr8 is only 6 bp away from Sanger sequencing result. Altogether, our analysis demonstrated that LongGF can detect gene fusions and infer relatively precise breakpoints using long-read RNA-seq data.

\section{Discussion}

Gene fusion is a well-known strategy used by cells to generate new genes in transcriptome, and many existing studies have found that some gene fusions contribute to the initiation or progression of different human cancer. Although short-read RNA-seq techniques provide a way to detect gene fusions for transcriptome data, long-read RNA-seq techniques represent theoretically better solutions to overcome the limitations of short-read techniques. However, it is not straightforward to detect gene fusions from noisy long-read RNA-seq data, and in this study, we propose LongGF to detect gene fusions from long-read RNA-seq data efficiently and effectively. LongGF is implemented in $\mathrm{C}++$ and is very fast to run, and it only takes several minutes and $<3 G B$ memory on 50,000 long reads from a transcriptome for gene fusion detection. Our evaluation also showed that LongGF can accurately detect true gene fusions on simulation datasets and four real datasets. Thus, LongGF is a useful tool for long-read RNA-seq data analysis, especially on cancer samples.

However, there are some limitations in LongGF. First, LongGF cannot detect gene fusions with unknown genes, since LongGF requires a pre-defined definition of all genes/exons in a GTF file. Therefore, this version of LongGF only detects candidate gene fusions from two known genes. To allow the detection of gene fusions involving novel genes/exons, users can modify the standard GTF file and include additional genes/exons. Second, LongGF may generate false positive predictions on gene fusions when dealing with homologous genes in the genome. That is, if several genes in a transcriptome share similar sequences (possibly part of the transcript sequence), it will be difficult to distinguish which gene the fused gene comes from. For example, if gene $\mathrm{A}$ and gene $\mathrm{B}$ have similar sequence and part of gene $\mathrm{A}$ is fused with part of gene $C$ for a hybrid gene $D$, it is hard to find whether the gene $\mathrm{D}$ is formed from gene $\mathrm{A}$ and gene $\mathrm{C}$, or gene $\mathrm{B}$ and gene $\mathrm{C}$. Third, LongGF may miss gene fusions from very short genes. In LongGF, we require an alignment is long enough to be significant and that an alignment has substantial overlap with a gene for further analysis. If only a smaller fraction $(<100 \mathrm{bp})$ of a gene is involved in a gene fusion, it is hard to distinguish the fusion candidates from sequencing/alignment noises. In LongGF, although users can set smaller thresholds to get gene fusions with smaller segments, they will generate more candidate fusion events and need to filter noisy candidates in the results.

With full-length transcriptome sequencing, we expect that long-read RNA-seq data (Oxford Nanopore and PacBio) will greatly facilitate gene fusion detection by overcoming many technical limitations of short reads. Compared to PacBio (either with traditional library or $\mathrm{HiFi}$ library preparation protocols), at fixed cost, Oxford Nanopore may be a more promising platform in gene fusion detection while generating data with higher error rate. This is because Nanopore currently has lower perbase cost of data generation, and our real data analysis showed that sequencing data with high read coverage can improve detection accuracy significantly. For Nanopore RNA-seq, there are two types: direct mRNA sequencing and cDNA sequencing. Compared to direct mRNA sequencing, cDNA sequencing allows samples to be amplified and requires less amount of starting materials, making it attractive in some cases. With more materials for sequencing (possibly in multiple flow cells), this can increase the read coverage at fusion breakpoint, and facilitate LongGF to detect gene fusions with low expression or low allele fraction (such as the BCR-ABL1 fusion discussed earlier). Additionally, we will conduct more comparison of the performance between LongGF with existing short read tools, for samples where both short-read and long-read sequencing data are available and the sample is available for experimental validation. We expect that we may find fusion events that are missed by short-read sequencing approaches, even when the sequencing coverage in short-read data is very high, if part of the fusion event falls under repetitive genomic regions.

\section{Conclusion}

In summary, LongGF is a fast and effective computational tool to detect candidate gene fusions from longread RNA-seq data. With the advancement of long-read sequencing techniques, we expect that LongGF will significantly contribute to the discovery of disease-causal gene fusions in the studies of human genetic diseases and cancer. 


\section{Abbreviations}

LongGF: Long-read gene fusion detector; UHR: Universal Human Reference RNA sample; AML: Acute myeloid leukemia

\section{Acknowledgements}

We thank the Wang lab members and anonymous reviewers for their insightful comments to improve the quality of the computational tool and the manuscript. We also thank Xuelian Chen and Ying Chen for the preparation of the AML samples for sequencing.

\section{About this supplement}

This article has been published as part of BMC Genomics Volume 21 Supplement 11 2020: Bioinformatics methods for biomedical data science. The full contents of the supplement are available at https://bmcgenomics. biomedcentral.com/articles/supplements/volume-21-supplement-11.

\section{Authors' contributions}

QL, KW and JFZ designed the study. QL implemented the tool and performed the evaluation on all real Nanopore and Pacbio data. YH conducted the simulations and evaluated the tool on the simulation data. AS and JFZ prepared the AML sample. LF conducted Nanopore sequencing and experimental validation of fusion breakpoints. KW supervised the study. $\mathrm{QL}, \mathrm{KW}$ and $\mathrm{YH}$ drafted and revised the manuscript. All authors read and approved the manuscript.

\section{Funding}

This study was supported by the NIH grants CA197903 to JZ and GM132713 to KW. Publication costs are funded by the NIH grants GM132713 to KW. The funding bodies play no role in the design of the study, data analysis, tool implementation and manuscript writing.

\section{Availability of data and materials}

The direct mRNA sequencing data on UHR and CDNA sequencing data on AML are available at NCBI Short Read Archive under BioProject accession PRJNA639366 and PRJNA640456, respectively. The LongGF software tool is available at https://github.com/WGLab/LongGF.

\section{Ethics approval and consent to participate}

Not applicable.

\section{Consent for publication}

Not applicable.

\section{Competing interests}

The authors declare that they have no competing interests.

\section{Author details}

'Raymond G. Perelman Center for Cellular and Molecular Therapeutics, Children's Hospital of Philadelphia, Philadelphia, PA 19104, USA. ²Department of Otolaryngology, Keck School of Medicine, University of Southern California, Los Angeles, CA 90033, USA. ${ }^{3}$ Department of Pathology and Laboratory Medicine, Perelman School of Medicine, University of Pennsylvania, Philadelphia, PA 19104, USA.

Received: 21 October 2020 Accepted: 29 October 2020 Published: 29 December 2020

\section{References}

1. Singh A, Zahra S, Das D, Kumar S. AtFusionDB: a database of fusion transcripts in Arabidopsis thaliana. Database (Oxford). 2019;2019.

2. National Academy of Sciences. Science (New York, NY). 1960;132(3438): 1488-501.

3. Mitelman F, Johansson B, Mertens F. The impact of translocations and gene fusions on cancer causation. Nat Rev Cancer. 2007;7(4):233-45.

4. Edwards PAW. Fusion genes and chromosome translocations in the common epithelial cancers. J Pathol. 2010;220(2):244-54.

5. Parker BC, Zhang W. Fusion genes in solid tumors: an emerging target for cancer diagnosis and treatment. Chin J Cancer. 2013;32(11):594-603.

6. Turc-Carel C, Philip I, Berger MP, Philip T, Lenoir G. Chromosomal translocation $(11 ; 22)$ in cell lines of Ewing's sarcoma. Comptes rendus des seances de l'Academie des sciences Serie III, Sciences de la vie. 1983; 296(23):1101-3.

7. Turc-Carel C, Dal Cin P, Limon J, Rao U, Li FP, Corson JM, Zimmerman R Parry DM, Cowan JM, Sandberg AA. Involvement of chromosome X in primary cytogenetic change in human neoplasia: nonrandom translocation in synovial sarcoma. Proc Natl Acad Sci U S A. 1987;84(7):1981-5.

8. Tomlins SA, Rhodes DR, Perner S, Dhanasekaran SM, Mehra R, Sun XW, Varambally S, Cao X, Tchinda J, Kuefer R, et al. Recurrent fusion of TMPRSS2 and ETS transcription factor genes in prostate cancer. Science (New York, NY). 2005;310(5748):644-8.

9. Tognon C, Knezevich SR, Huntsman D, Roskelley CD, Melnyk N, Mathers JA, Becker L, Carneiro F, MacPherson N, Horsman D, et al. Expression of the ETV6-NTRK3 gene fusion as a primary event in human secretory breast carcinoma. Cancer Cell. 2002;2(5):367-76.

10. Williams SV, Hurst CD, Knowles MA. Oncogenic FGFR3 gene fusions in bladder cancer. Hum Mol Genet. 2013;22(4):795-803.

11. Seshagiri S, Stawiski EW, Durinck S, Modrusan Z, Storm EE, Conboy CB, Chaudhuri S, Guan Y, Janakiraman V, Jaiswal BS, et al. Recurrent R-spondin fusions in colon cancer. Nature. 2012:488(7413):660-4.

12. Salzman J, Marinelli RJ, Wang PL, Green AE, Nielsen JS, Nelson BH, Drescher CW, Brown PO. ESRRA-C11orf20 is a recurrent gene fusion in serous ovarian carcinoma. PLoS Biol. 2011;9(9):e1001156.

13. Soda M, Choi YL, Enomoto M, Takada S, Yamashita Y, Ishikawa S, Fujiwara S, Watanabe $\mathrm{H}$, Kurashina K, Hatanaka $\mathrm{H}$, et al. Identification of the transforming EML4-ALK fusion gene in non-small-cell lung cancer. Nature. 2007:448(7153):561-6.

14. Parker BC, Annala MJ, Cogdell DE, Granberg KJ, Sun Y, Ji P, Li X, Gumin J, Zheng $\mathrm{H}$, Hu L, et al. The tumorigenic FGFR3-TACC3 gene fusion escapes miR-99a regulation in glioblastoma. J Clin Invest. 2013;123(2):855-65.

15. Jones DT, Kocialkowski S, Liu L, Pearson DM, Backlund LM, Ichimura K Collins VP. Tandem duplication producing a novel oncogenic BRAF fusion gene defines the majority of pilocytic astrocytomas. Cancer Res. 2008;68(21):8673-7.

16. Ariazi EA, Clark GM, Mertz JE. Estrogen-related receptor a and estrogenrelated receptor $y$ associate with unfavorable and favorable biomarkers, respectively, in human breast cancer. Cancer Res. 2002;62(22):6510-8.

17. Sun P, Sehouli J, Denkert C, Mustea A, Könsgen D, Koch I, Wei L, Lichtenegger W. Expression of estrogen receptor-related receptors, a subfamily of orphan nuclear receptors, as new tumor biomarkers in ovarian cancer cells. J Mol Med. 2005;83(6):457-67.

18. Göransson M, Andersson MK, Forni C, Ståhlberg A, Andersson C, Olofsson A, Mantovani R, Åman P. The myxoid liposarcoma FUS-DDIT3 fusion oncoprotein deregulates NF-KB target genes by interaction with NFKBIZ. Oncogene. 2009;28(2):270-8.

19. Olmos D, Martins AS, Jones RL, Alam S, Scurr M, Judson IR. Targeting the insulin-like growth factor 1 receptor in Ewing's sarcoma: reality and expectations. Sarcoma. 2011;2011.

20. Scotlandi K, Benini S, Sarti M, Serra M, Lollini P-L, Maurici D, Picci P, Manara $M C$, Baldini N. Insulin-like growth factor I receptor-mediated circuit in Ewing's sarcoma/peripheral neuroectodermal tumor: a possible therapeutic target. Cancer Res. 1996;56(20):4570-4.

21. Wu Y-M, Su F, Kalyana-Sundaram S, Khazanov N, Ateeq B, Cao X, Lonigro RJ, Vats $P$, Wang $R$, Lin $S$-F. Identification of targetable FGFR gene fusions in diverse cancers. Cancer Discov. 2013;3(6):636-47.

22. Uhrig S, Fröhlich M, Hutter B, Brors B. PO-400 Arriba - fast and accurate gene fusion detection from rna-seq data, vol. 3; 2018.

23. Iyer MK, Chinnaiyan AM, Maher CA. ChimeraScan: a tool for identifying chimeric transcription in sequencing data. Bioinformatics (Oxford, England). 2011;27(20):2903-4.

24. Rodriguez-Martin B, Palumbo E, Marco-Sola S, Griebel T, Ribeca P, Alonso G, Rastrojo A, Aguado B, Guigo R, Djebali S. ChimPipe: accurate detection of fusion genes and transcription-induced chimeras from RNA-seq data. BMC Genomics. 2017;18(1):7.

25. McPherson A, Hormozdiari F, Zayed A, Giuliany R, Ha G, Sun MG, Griffith M, Heravi Moussavi A, Senz J. Melnyk N et al: deFuse: an algorithm for gene fusion discovery in tumor RNA-Seq data. PLoS Comput Biol. 2011;7(5): e1001138.

26. Nicorici $D$, Satalan $M$, Edgren $H$, Kangaspeska S, Murumägi A, Kallioniemi $O$, Virtanen S, Kilkku O. FusionCatcher - a tool for finding somatic fusion genes in paired-end RNA-sequencing data. bioRxiv. 2014;011650. 
27. Li Y, Chien J, Smith DI, Ma J. FusionHunter: identifying fusion transcripts in cancer using paired-end RNA-seq. Bioinformatics (Oxford, England). 2011; 27(12):1708-10.

28. Ge H, Liu K, Juan T, Fang F, Newman M, Hoeck W. FusionMap: detecting fusion genes from next-generation sequencing data at base-pair resolution. Bioinformatics (Oxford, England). 2011;27(14):1922-8.

29. Liu C, Ma J, Chang CJ, Zhou X. FusionQ: a novel approach for gene fusion detection and quantification from paired-end RNA-Seq. BMC bioinformatics. 2013;14:193.

30. Kim P, Jang YE, Lee $S$. FusionScan: accurate prediction of fusion genes from RNA-Seq data. Genomics \& informatics. 2019;17(3):e26.

31. Okonechnikov K, Imai-Matsushima A, Paul L, Seitz A, Meyer TF, GarciaAlcalde F. InFusion: advancing discovery of fusion genes and chimeric transcripts from deep RNA-sequencing data. PLoS One. 2016;11(12): e0167417.

32. Wang K, Singh D, Zeng Z, Coleman SJ, Huang Y, Savich GL, He X, Mieczkowski P, Grimm SA, Perou CM, et al. MapSplice: accurate mapping of RNA-seq reads for splice junction discovery. Nucleic Acids Res. 2010;38(18):e178.

33. Torres-Garcia W, Zheng S, Sivachenko A, Vegesna R, Wang Q, Yao R, Berger MF, Weinstein JN, Getz G, Verhaak RG. PRADA: pipeline for RNA sequencing data analysis. Bioinformatics (Oxford, England). 2014;30(15):2224-6.

34. Asmann YW, Hossain A, Necela BM, Middha S, Kalari KR, Sun Z, Chai HS, Williamson DW, Radisky D, Schroth GP, et al. A novel bioinformatics pipeline for identification and characterization of fusion transcripts in breast cancer and normal cell lines. Nucleic Acids Res. 2011;39(15):e100.

35. Jia W, Qiu K, He M, Song P, Zhou Q, Zhou F, Yu Y, Zhu D, Nickerson ML, Wan $\mathrm{S}$, et al. SOAPfuse: an algorithm for identifying fusion transcripts from paired-end RNA-Seq data. Genome Biol. 2013;14(2):R12.

36. Haas BJ, Dobin A, Li B, Stransky N, Pochet N, Regev A. Accuracy assessment of fusion transcript detection via read-mapping and de novo fusion transcript assembly-based methods. Genome Biol. 2019;20(1):213.

37. Akers NK, Schadt EE, Losic B. STAR Chimeric Post for rapid detection of circular RNA and fusion transcripts. Bioinformatics (Oxford, England). 2018; 34(14):2364-70.

38. Kim D, Salzberg SL. TopHat-fusion: an algorithm for discovery of novel fusion transcripts. Genome Biol. 2011;12(8):R72

39. Chen K, Wallis JW, Kandoth C, Kalicki-Veizer JM, Mungall KL, Mungall AJ, Jones SJ, Marra MA, Ley TJ, Mardis ER, et al. BreakFusion: targeted assemblybased identification of gene fusions in whole transcriptome paired-end sequencing data. Bioinformatics (Oxford, England). 2012;28(14):1923-4.

40. Benelli M, Pescucci C, Marseglia G, Severgnini M, Torricelli F, Magi A. Discovering chimeric transcripts in paired-end RNA-seq data by using EricScript. Bioinformatics (Oxford, England). 2012;28(24):3232-9.

41. Chiu R, Nip KM, Birol I. Fusion-Bloom: fusion detection in assembled transcriptomes. Bioinformatics (Oxford, England). 2019;36:2256.

42. Vu TN, Deng W, Trac QT, Calza S, Hwang W, Pawitan Y. A fast detection of fusion genes from paired-end RNA-seq data. BMC Genomics. 2018;19(1):786.

43. Davidson NM, Majewski IJ, Oshlack A. JAFFA: high sensitivity transcriptomefocused fusion gene detection. Genome Med. 2015;7(1):43.

44. Fotakis G, Rieder D, Haider M, Trajanoski Z, Finotello F. NeoFuse: predicting fusion neoantigens from RNA sequencing data. Bioinformatics (Oxford, England). 2019;36:2260.

45. McPherson A, Wu C, Wyatt AW, Shah S, Collins C, Sahinalp SC. nFuse: discovery of complex genomic rearrangements in cancer using highthroughput sequencing. Genome Res. 2012;22(11):2250-61.

46. Melsted P, Hateley S, Joseph IC, Pimentel H, Bray N, Pachter L. Fusion detection and quantification by pseudoalignment. bioRxiv. 2017;166322.

47. Kinsella M, Harismendy $O$, Nakano M, Frazer KA, Bafna V. Sensitive gene fusion detection using ambiguously mapping RNA-Seq read pairs. Bioinformatics (Oxford, England). 2011;27(8):1068-75.

48. Kumar S, Vo AD, Qin F, Li H. Comparative assessment of methods for the fusion transcripts detection from RNA-Seq data. Sci Rep. 2016:6: 21597.

49. Liu S, Tsai WH, Ding Y, Chen R, Fang Z, Huo Z, Kim S, Ma T, Chang TY, Priedigkeit NM, et al. Comprehensive evaluation of fusion transcript detection algorithms and a meta-caller to combine top performing methods in paired-end RNA-seq data. Nucleic Acids Res. 2016;44(5):e47.

50. Byrne A, Beaudin AE, Olsen HE, Jain M, Cole C, Palmer T, DuBois RM, Forsberg EC, Akeson M, Vollmers C. Nanopore long-read RNAseq reveals widespread transcriptional variation among the surface receptors of individual B cells. Nat Commun. 2017;8:16027.

51. Tang AD, Soulette CM, van Baren MJ, Hart K, Hrabeta-Robinson E, Wu CJ, Brooks AN. Full-length transcript characterization of SF3B1 mutation in chronic lymphocytic leukemia reveals downregulation of retained introns. Nat Commun. 2020;11(1):1438.

52. Hu Y, Fang L, Chen X, Zhong JF, Li M, Wang K: LIQA: Long-read Isoform Quantification and Analysis. bioRxiv 2020.

53. Li H: Minimap2: pairwise alignment for nucleotide sequences. Bioinformatics (Oxford, England) 2018, 34(18):3094-3100.

54. Consortium M, Shi L, Reid LH, Jones WD, Shippy R, Warrington JA, Baker SC, Collins PJ, de Longueville F, Kawasaki ES, et al. The MicroArray quality control (MAQC) project shows inter- and intraplatform reproducibility of gene expression measurements. Nat Biotechnol. 2006;24(9):1151-61.

55. Shi L, Campbell G, Jones WD, Campagne F, Wen Z, Walker SJ, Su Z, Chu TM, Goodsaid FM, Pusztai L, et al. The MicroArray quality control (MAQC)-II study of common practices for the development and validation of microarraybased predictive models. Nat Biotechnol. 2010;28(8):827-38.

56. Gao F, Kim JM, Kim J, Lin M-Y, Liu CY, Russin JJ, Walker CP, Dominguez R, Camarena A, Nguyen JD. Evaluation of biological and technical variations in low-input RNA-Seq and single-cell RNA-Seq. Int J Comp Biol Drug Design. 2018:11(1-2):5-22

57. Xu J, Su Z, Hong H, Thierry-Mieg J, Thierry-Mieg D, Kreil DP, Mason CE, Tong W, Shi L. Cross-platform ultradeep transcriptomic profiling of human reference RNA samples by RNA-Seq. Sci Data. 2014;1:140020.

58. Garcia-Alonso L, Holland CH, Ibrahim MM, Turei D, Saez-Rodriguez J. Benchmark and integration of resources for the estimation of human transcription factor activities. Genome Res. 2019;29(8):1363-75.

59. Teng M, Love MI, Davis CA, Djebali S, Dobin A, Graveley BR, Li S, Mason CE, Olson S, Pervouchine D, et al. A benchmark for RNA-seq quantification pipelines. Genome Biol. 2016;17:74

60. Hu Y, Liu Y, Mao X, Jia C, Ferguson JF, Xue C, Reilly MP, Li H, Li M. PennSeq: accurate isoform-specific gene expression quantification in RNA-Seq by modeling non-uniform read distribution. Nucleic Acids Res. 2014;42(3):e20.

61. Hayer KE, Pizarro A, Lahens NF, Hogenesch JB, Grant GR. Benchmark analysis of algorithms for determining and quantifying full-length mRNA splice forms from RNA-seq data. Bioinformatics. 2015;31(24):3938-45.

62. Sequel II System Data Release: Universal Human Reference (UHR) Iso Seq [https://github.com/PacificBiosciences/DevNet/wiki/Sequel-II-System-DataRelease:-Universal-Human-Reference-(UHR)-Iso-Seq]; Accessed on 10 Jun 2020.

63. Yang C, Chu J, Warren RL, Birol I. NanoSim: nanopore sequence read simulator based on statistical characterization. Gigascience. 2017;6(4):1-6.

64. Mitelman F, Johansson B, Mertens F. Mitelman Database of Chromosome Aberrations and Gene Fusions in Cancer; 2020

65. Atlas of Genetics and Cytogenetics in Oncology and Haematology [http:// atlasgeneticsoncology.org/Genes/GC_B3GAT3.html]; Accessed on 10 Jun 2020

66. Weirather JL, Afshar PT, Clark TA, Tseng E, Powers LS, Underwood JG, Zabner J, Korlach J, Wong WH, Au KF. Characterization of fusion genes and the significantly expressed fusion isoforms in breast cancer by hybrid sequencing. Nucleic Acids Res. 2015:43(18):e116.

67. Lan F, Shi Y. Histone H3.3 and cancer: a potential reader connection. Proc Natl Acad Sci U S A. 2015;112(22):6814-9.

68. Lytovchenko O, Kunji ERS. Expression and putative role of mitochondrial transport proteins in cancer. Biochim Biophys Acta Bioenerg. 2017;1858(8): 641-54.

69. Vandepoele K, Van Roy N, Staes K, Speleman F, Van Roy F. A novel gene family NBPF: intricate structure generated by gene duplications during primate evolution. Mol Biol Evol. 2005;22(11):2265-74.

70. Obayashi S, Horiguchi J, Higuchi T, Katayama A, Handa T, Altan B, Bai T, Bao $\mathrm{P}$, Bao H, Yokobori T, et al. Stathmin1 expression is associated with aggressive phenotypes and cancer stem cell marker expression in breast cancer patients. Int J Oncol. 2017;51(3):781-90.

71. Luo Y, Kong F, Wang Z, Chen D, Liu Q, Wang T, Xu R, Wang X, Yang JY. Loss of ASAP3 destabilizes cytoskeletal protein ACTG1 to suppress cancer cell migration. Mol Med Rep. 2014;9(2):387-94.

72. Ohki M. Molecular basis of the $t(8 ; 21)$ translocation in acute myeloid leukaemia. Semin Cancer Biol. 1993;4(6):369-75.

\section{Publisher's Note}

Springer Nature remains neutral with regard to jurisdictional claims in published maps and institutional affiliations. 